\title{
C-peptide prevents SMAD3 binding to alpha promoters to inhibit collagen type IV synthesis
}

\author{
Yanning Li1,*, Yan Zhong2,*, Wenjian Gong1, Xuehan Gao1, Huanli Qi², Kun Liu² and Jinsheng Qi² \\ 1Department of Molecular Biology, Hebei Key Lab of Laboratory Animal Science, Hebei Medical University, Shijiazhuang, China \\ 2Department of Biochemistry, Hebei Key Laboratory of Medical Biotechnology, Hebei Medical University, Shijiazhuang, China \\ Correspondence should be addressed to J Qi: qijinsheng777@163.com \\ *(Y Li and Y Zhong contributed equally to this work)
}

\begin{abstract}
Activation of transforming growth factor $\beta 1$ (TGFB1)/SMAD3 signaling may lead to additional synthesis of collagen type IV (COL4), which is a major contributor to extracellular matrix (ECM) accumulation in diabetic nephropathy (DN). C-peptide can attenuate fibrosis to have unique beneficial effects in DN. However, whether and how C-peptide affects TGFB1/SMAD3-activated COL4 synthesis is unclear. In this study, pathological changes, expression of COL4 a1-a5 chains (Col4a1-a5), COL4 distribution and protein and TGFB1 and SMAD3 protein were first assessed in a rat model of diabetes. Then, rat mesangial cells were treated with high glucose (HG) and/or C-peptide to investigate the underlying mechanism. Col4a1-a5 expression, COL4 protein and secretion, TGFB1 protein, SMAD3 nuclear translocation and binding of SMAD3 to its cognate sites in the promoters of Col4a1a2, Col4a3a4 and Col4a5 were measured. It was found that C-peptide attenuated glomerular pathological changes and suppressed renal Col4a1-a5 mRNA expression, COL4 protein content and TGFB1 protein content. C-peptide had a dose-dependent effect to inhibit Col4a1-a5 mRNA expression, COL4 protein content and secretion, in HG-stimulated mesangial cells. In addition, the HG-induced increase in TGFB1 protein content was significantly reduced by C-peptide. Although not apparently affecting SMAD3 nuclear translocation, C-peptide prevented SMAD3 from binding to its sites in the Col4a1a2, Col4a3a4 and Col4a5 promoters in HG-stimulated mesangial cells. In conclusion, C-peptide could prevent SMAD3 from binding to its sites in the Col4a1a2, Col4a3a 4 and Col4a5 promoters, to inhibit COL4 generation. These results may provide a mechanism for the alleviation of fibrosis in DN by C-peptide.
\end{abstract}

$\begin{array}{ll} & \text { Key Words } \\ \text { - } & \text { C-peptide } \\ \text { - } & \text { collagen type IV } \\ \text { - TGFB1 } \\ \text { - SMAD3 }\end{array}$

Journal of Molecular Endocrinology (2018) 61, 47-56

\section{Introduction}

Diabetic nephropathy (DN), a serious microvascular complication of diabetes, remains the leading cause of chronic kidney disease and end-stage renal disease (Fan et al. 2017, Flyvbjerg 2017). Excess extracellular matrix (ECM) deposition, leading to glomerular basement membrane thickening, mesangial expansion and then glomerulosclerosis, is a feature of DN (Kato et al. 2016, Marshall 2016). Mesangial cells are believed to be a major source of ECM, and their dysfunction may lead to ECM accumulation in DN (Donegan et al. 2016, Sheu et al. 2017). The underlying mechanism of this ECM deposition is under active investigation (Al-Waili et al. 2017). 
Transforming growth factor $\beta 1$ (TGFB1)/SMAD signaling is the key pathway that promotes fibrosis in DN (Chung et al. 2010, Gao et al. 2016). In diabetes, TGFB1 binds to its receptor, which activates downstream signaling, especially via SMAD3, to promote renal fibrosis (Chung et al. 2010). It has been shown that TGFB1 can trigger excess synthesis of collagen type IV (COL4), which is the most abundant protein in ECM (Turner et al. 2015, Peng et al. 2016, Dimas et al. 2017). COL4 has a triple -helical structure composed of three a chains, which are encoded by six genes (Robertson et al. 2014, Sugiyama et al. 2015). The genes expressing a1-a6 have two lines head to head, such that a1 and a2 share the same promoter, as do a3 and a4, and a5 and a6. The established COL4 heterotrimers are a1a2a1 and a3a4a5, and the predicted SMAD3-binding sites have also been found in the Col4a1a2, Col4a3a4 and Col4a5 promoters (Robertson et al. 2014). Although excess TGFB1/SMAD3stimulated COL4 synthesis has been established to be a major contributor to ECM accumulation in DN, there is still no effective therapy.

C-peptide links the A and B chains of proinsulin, but has been found to have unique beneficial effects on DN (Shaw et al. 2015, Wahren \& Larsson 2015). In animal models or in humans with type 1 diabetes, C-peptide can reduce glomerular hyperfiltration and albuminuria (Brunskill 2017). When administered to diabetic rodents in physiological concentrations, C-peptide attenuates mesangial expansion and ECM accumulation (Wahren 2017). The underlying mechanisms of its protective effects have not been fully elucidated, although various candidate mechanisms have been proposed (Lindahl et al. 2010, Garcia-Serrano et al. 2015, Kolar et al. 2017). However, these mechanisms are not specific to DN and do not account for the unique effect of $\mathrm{C}$-peptide to attenuate ECM accumulation in DN. We have previously shown that C-peptide dynamically localizes to the nucleus in high glucose (HG)-stimulated mesangial cells, providing a model to further investigate these protective mechanisms (Li et al. 2013b). TGFB1 signaling, the major pro-fibrotic stimulus, has been found to be indirectly antagonized by C-peptide (Hills et al. 2010a,b). However, the effects of C-peptide on excess TGFB1/SMAD3-stimulated COL4 synthesis in $\mathrm{DN}$ are unknown.

In this study, the effects of C-peptide on pathological changes, Col4a1-a5 mRNA expression and COL4 protein, and TGFB1 and SMAD3 protein, were first assessed in a rat model of diabetes. Then, the effects of C-peptide on Col4a1-a5 mRNA expression, COL4 protein and secretion, TGFB1 and thrombospondin-1 (TSP-1) proteins, SMAD3 nuclear translocation and binding of SMAD3 to its sites in the Col4a1a2, Col4a3a4 and Col4a5 promoters were measured in HG-stimulated mesangial cells.

\section{Materials and methods}

\section{Animals}

A total of 40 healthy male Sprague-Dawley rats (252.05 $\pm 27.17 \mathrm{~g}$, about 2 months old) were provided by the Department of Experimental Animals of Hebei Medical University. Experiment protocol followed the guidelines established by the Ethics Review Committee for Animal Experimentation of Hebei Medical University. Rats were randomly divided into three groups: diabetes mellitus (DM) group $(n=15), \mathrm{DM}+\mathrm{C}$-peptide $(\mathrm{DM}+\mathrm{CP})$ group $(n=15)$ and control (Con) group $(n=10)$. The former two groups were injected intraperitoneally with streptozotocin ( $40 \mathrm{mg} / \mathrm{kg}$, dissolved in $0.1 \mathrm{~mol} / \mathrm{L}$ citrate buffer, $\mathrm{pH} 4.4$, freshly made, $10 \mathrm{mg} / \mathrm{mL}$ ), to induce diabetes. Rats in $\mathrm{DM}+\mathrm{CP}$ group were injected subcutaneously twice daily with $130 \mathrm{nmol} / \mathrm{kg}$ C-peptide (Ido et al. 1997, Sun et al. 2010), and other rats were given equivalent saline. Body mass and fasting blood glucose were measured at 3 days and 12 weeks of established diabetes. At 12 weeks, renal cortex was collected for hematoxylin-eosin (HE) staining, and a segment of renal cortex was used for detections of Col4a1-a5 mRNA expressions, COL4 protein and immunohistochemical staining and TGFB1 and SMAD3 protein.

\section{Cells and treatments}

Rat mesangial cells (HBZY-1) were obtained from China Center for Type Culture Collection and cultured in DMEM containing $5 \mathrm{mM}$ glucose and 10\% fetal bovine serum. Cells were treated with 20, 25, 30, 35 and $40 \mathrm{mM}$ $\mathrm{HG}$ for $24 \mathrm{~h}$, and then $35 \mathrm{mM}$ glucose plus $0.1,0.5$ and $0.9 \mathrm{nM}$ C-peptide for $24 \mathrm{~h}$, based on our previous study (Li et al. 2013b). Cells were collected for detection of Col4a1-a5 mRNA expression. And after 48-h treatment, cells were collected for detection of COL 4 protein content and culture medium for detection of COL4 secretion. Then, cells were divided into low glucose (LG) group (5 mM glucose), $\mathrm{HG}$ group (35 mM glucose) and $\mathrm{CP}$ group (35 mM glucose +0.5 nM C-peptide). High L-glucose (L-HG) with the concentration of $35 \mathrm{mM}$ L-glucose was used as a hypertonic control. After cells were pretreated with HG and subsequent HG plus C-peptide for $24 \mathrm{~h}$, SMAD3 nuclear translocation and binding of SMAD3 to 
its sites in the Col4a1a2, Col4a3a4 and Col4a5 promoters were measured. The protein contents of TGFB1, TSP-1 and SMAD3 were evaluated after 48 -h treatments. In addition, the localization of C-peptide was detected.

\section{Quantitative real-time RT-PCR}

Total RNA was isolated using TRIzol reagent (TaKaRa) and reverse transcribed into cDNA using RevertAid First Strand cDNA synthesis Kit (Fermentas, Shanghai, China), followed by real-time PCR amplification using specific primers (Table 1). Actin primers were used as an internal standard. The comparative $\mathrm{Ct}$ method was used to calculate the relative abundance of the mRNA compared with that of actin.

\section{Immunohistochemistry}

Distribution and content of glomerular COL4 were detected by immunohistochemistry. The procedures were as follows: renal cortex embedded in paraffin was cut and dewaxed. After incubated with $\mathrm{H}_{2} \mathrm{O}_{2}$, sections were incubated with goat serum. Sections were incubated with anti-COL4 antibody (diluted 1:50, Bioworld, Nanjing, China) and then the secondary antibody (diluted 1:200, Zhongshan, Beijing, China). 3, 3 -Diaminobenzidine (DAB) solution was used for color emergence. The mean density of ten glomeruli from three rats in each group was quantified by Image Pro Plus 6.0.

\section{Enzyme-linked immunosorbent assay}

After treatment, the culture medium was collected to analyze COL4 secretion by ELISA (Jiancheng, Nanjing, China). Standards and samples were added to wells of the plate and incubated for $1 \mathrm{~h}$. After the wells were washed with ELISA wash buffer, the conjugated antibody was added and incubated for $1 \mathrm{~h}$. Then, the wells were washed again with the ELISA wash buffer. The substrate was added in the wells and incubated for $15 \mathrm{~min}$. Then, the stop solution was added and absorption was measured with an ELISA reader at $450 \mathrm{~nm}$. The tests were carried out in duplicate.

\section{Western blotting}

The protein contents of COL4, TGFB1, TSP-1, total and nuclear SMAD3 and phospho-SMAD3 were detected with the protocol previously described (Li et al. 2013a). The anti-COL4, anti-TGFB1 and anti-TSP-1 antibodies (diluted 1:200, Bioworld), anti-SMAD3 antibody (diluted 1:500, CST, Shanghai, China) or anti-phospho-SMAD3 antibody (diluted 1:100, Bioworld) was used. Band intensity was quantified and calculated. Actin routinely served as a loading control.

\section{Immunofluorescence}

The localization of C-peptide and the nuclear translocation of SMAD3 were detected by immunofluorescence with the previously reported protocol (Li et al. 2013a). The used primary antibody was anti-CP (diluted 1:2000, Abcam, Shanghai, China), anti-SMAD3 (diluted 1:100, CST) and the secondary antibody was FITC conjugated (diluted 1:200, Zhongshan). The images of C-peptide were taken with a fluorescence microscope (Olympus IX51), and the images of SMAD3 were taken with a laser confocal scanning microscope (Olympus). Nuclear SMAD3-positive cells from ten visual fields at random in each group were recorded. The percentage of SMAD3 nuclear translocation was calculated. To get the exact results, controls (PBS instead of the first antibody or the second antibody) were designed.

\section{Chromatin immunoprecipitations}

The predicted SMAD3-binding sites in the shared Col4a1a2, Col4a3a4 and Col4a5 promoters were shown in

Table 1 Primers used for real-time PCR detection.

\begin{tabular}{|c|c|c|c|c|}
\hline \multirow[b]{2}{*}{ Genes } & \multicolumn{2}{|c|}{ Primers } & \multirow{2}{*}{$\begin{array}{c}\text { Annealing } \\
\text { temperature }\left({ }^{\circ} \mathrm{C}\right)\end{array}$} & \multirow[b]{2}{*}{ Product size $(b p)$} \\
\hline & Sense & Anti-sense & & \\
\hline Col4a1 & 5' GTGTCAGCAATTAGGCAGGTCAAG 3' & 5' AGTCAGTCATTCAGTCTGCGGATAG 3' & 65 & 305 \\
\hline Col4a2 & 5' CTTCCGTGCAACACCATTTATCG 3' & 5' GGGTCGGCATCTGAGGTAATAAG 3' & 65 & 445 \\
\hline Col4a3 & 5' GAGACAGAGGGACACCTGCCAAC 3' & 5' TGGCATTGTGGTGAATCGCTG 3' & 63 & 209 \\
\hline Col4a4 & 5' CTGGTATGGACGGCTACAATG 3' & 5' CCCTAAATGCTCTGGTTCTGC 3' & 63 & 262 \\
\hline Col4a5 & 5' GATTCACAAGTACCTCAGCGATG 3' & 5' TGGGGGTACATTAGGATTAGTAGC 3' & 64 & 246 \\
\hline$\beta$-Actin & 5' CCGTAAAGACCTCTATGCCAAC 3' & 5' GTCAAAGAAAGGGTGTAAAACGC 3' & 60 & 303 \\
\hline
\end{tabular}

AT, annealing temperature. 
Supplementary Fig. 2 (see section on supplementary data given at the end of this article). Binding of SMAD3 to its sites was analyzed by chromatin immunoprecipitation (ChIP), performed as reported (Li et al. 2015). Briefly, antiSMAD3 antibody (CST) or control rabbit IgG (Santa Cruz) was added, and then protein G-agarose beads (Santa Cruz) were used. Then SMAD3-binding sites in the rat Col4a1a2, Col4a3a4 and Col4a5 promoters were amplified by realtime PCR, respectively. Primers sequences used for the binding sites were in Table 2 .

\section{DNA pull-down assay}

The contents of SMAD3 bound to the Col4a1a2, Col4a3a4 and Col4a5 promoters were measured by a DNA pulldown assay, as previously reported (Li et al. 2017). The sequences of the Col4a1a2 (Gene ID: 290905 and 306628), Col4a3a4 (Gene ID: 363265 and 301562) and Col4a5 (Gene ID: 363457) promoters were found. The oligonucleotides containing biotin on the 5 -end of Col4a1a2 promoter were biotin-5'-GGGGCCCCGGCTC AGCGTCTGGCTTCTGCTGCCGCTCGCC-3' (forward) and biotin-5'-GGCGAGCGGCAGCAGAAGCCAGACGC TGAGCCGGGGCCCC-3' (reverse). The oligonucleotides containing biotin on the $5^{\prime}$-end of Col4a3a4 promoter were biotin-5'-ACCCGGCCTCCAGAGTCGCAGACTCC CGCGCGCCTCCCGC-3' (forward) and biotin-5'-GCG GGAGGCGCGCGGGAGTCTGCGACTCTGGAGGCCG GGT-3' (reverse). The oligonucleotides containing biotin on the $5^{\prime}$-end of Col4a5 promoter were biotin-5'-AATA GTCCTAGCTCAGGTCTGTTTGTCAAAGGCTTTGATG-3' (forward) and biotin-5'-CATCAAAGCCTTTGACAAAC AGACCTGAGCTAGGACTATT-3' (reverse). The mutant oligonucleotides were shown in Supplementary Table 1. Each pair of oligonucleotides was annealed following standard protocols. Nuclear protein extracts were precleared with ImmunoPure streptavidin-agarose beads (Thermo, Shanghai, China), and the supernatant was incubated with $4 \mu \mathrm{g}$ of biotinylated double-stranded oligonucleotides. Twenty microliters of streptavidinagarose beads were added and the protein-DNAstreptavidin-agarose complex was washed, separated and subjected to western blotting with anti-SMAD3 antibody (CST).

\section{Statistical analysis}

Statistical analysis was performed using SPSS 17.0 software. Data were presented as mean \pm S.D. One-way ANOVA was used to analyze the differences among groups, and the differences between two groups were evaluated by the least-significant difference. Statistical significant was set at $P<0.05$.

\section{Results}

\section{C-peptide attenuates glomerular ECM accumulation in $D N$}

First, body mass and blood glucose were measured in the rats after 3 days and 12 weeks of established diabetes. Although there was no difference among the groups at the beginning of the experiment (Fig. 1A), body mass was significantly lower at 12 weeks in the $\mathrm{DM}$ and $\mathrm{DM}+\mathrm{CP}$ groups than in the Con group (Fig. 1B). Compared with the Con group, blood glucose was significantly higher throughout the experiment in the $\mathrm{DM}$ and $\mathrm{DM}+\mathrm{CP}$ groups (Fig. 1C and D). C-peptide treatment had no obvious effect on body mass and blood glucose of the diabetic rats.

Typical glomerular changes were demonstrated by HE staining (Fig. 1E). Pathological changes were not observed in the Con group. Nephrosclerosis and broadened capsular spaces were observed in the DM group, but glomerular pathological changes were observed to a considerably lesser extent in the $\mathrm{DM}+\mathrm{CP}$ group. These results indicate that C-peptide treatment could attenuate glomerular pathological changes in DN, without affecting body mass and blood glucose.

\section{C-peptide suppresses renal COL4 synthesis in diabetic rats}

Col4a1-a5 mRNA expression, and COL4 protein and distribution, were then evaluated after 12-week C-peptide

Table 2 Primers used for real-time PCR detection of Col IV gene promoters.

\begin{tabular}{|c|c|c|c|c|}
\hline \multirow[b]{2}{*}{ Gene promoters } & \multicolumn{2}{|c|}{ Primers } & \multirow{2}{*}{$\begin{array}{c}\text { Annealing } \\
\text { temperature }\left({ }^{\circ} \mathrm{C}\right)\end{array}$} & \multirow{2}{*}{$\begin{array}{l}\text { Product } \\
\text { size }(b p)\end{array}$} \\
\hline & Sense & Anti-sense & & \\
\hline Col4a1a2 & 5' GACTCCCTGCGACACCAAGTCC 3' & 5' GTGGCTCTGAGCAGAGACCTGAG 3' & 67 & 168 \\
\hline Col4a3a4 & 5' AAGACCGGCAGCAGATGGGCAG 3' & 5' GACTCCGGTGCCTAGCGGGTGC 3' & 68 & 189 \\
\hline Col4a5 & 5' CTATGTGACATGTGTTGTAACATGC 3' & 5' AGCGGATGGATTTCAGAGTGTG 3' & 60 & 230 \\
\hline
\end{tabular}



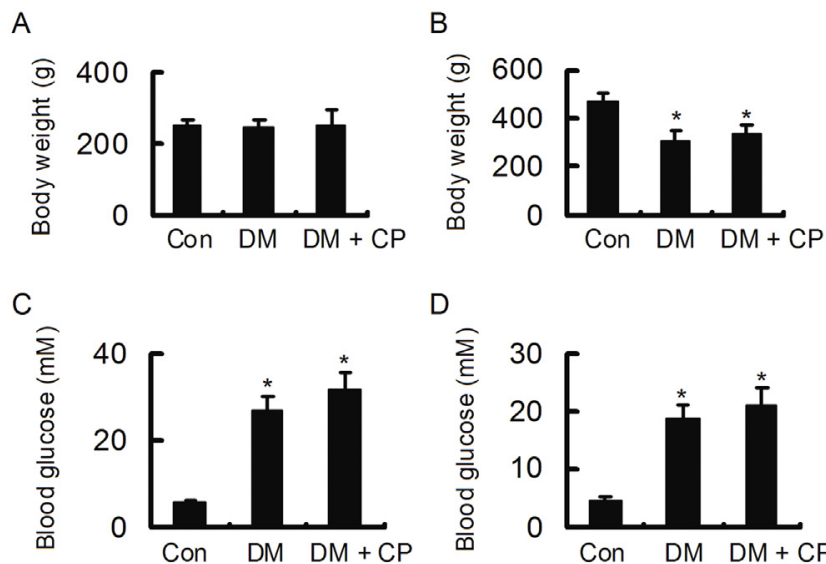

D

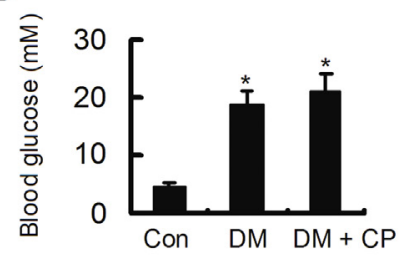

E

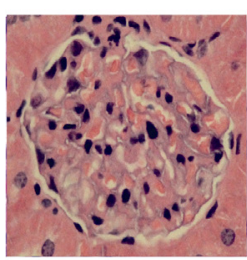

Con

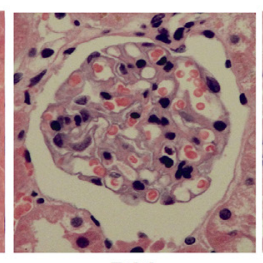

$\mathrm{DM}$

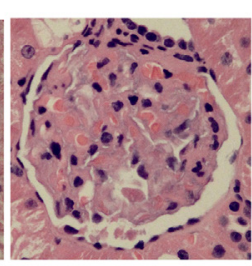

$\mathrm{DM}+\mathrm{CP}$

\section{Figure 1}

Effects of CP on pathological changes of diabetic nephropathy. Body weight at 3 days (A) and 12 weeks (B) of established diabetes were measured. Blood glucose at 3 days $(C)$ and 12 weeks (D) were also detected $(n=9)$. ${ }^{*} P<0.05$ vs control (Con) group. (E) HE staining of glomerulus. CP, C-peptide; DM, diabetes mellitus; $\mathrm{HE}$, hematoxylin and eosin.

treatment in the diabetic rats. Compared with the Con group, mRNA expressions of all of Col4a1-a5 were $4.69 \pm 0.72, \quad 4.22 \pm 0.65, \quad 4.11 \pm 0.55, \quad 3.94 \pm 0.54$ and $4.19 \pm 0.42$-fold, considerably higher in the DM group, but were $2.72 \pm 0.51,2.32 \pm 0.38,2.02 \pm 0.40,1.83 \pm 0.42$ and $2.14 \pm 0.60$-fold in the $\mathrm{DM}+\mathrm{CP}$ group, which were significantly reduced $(n=3, P<0.05)$ (Fig. 2A). Compared with the Con group, the $1.61 \pm 0.21$-fold increase in COL4 protein content in the DM group, however, was $1.13 \pm 0.15$ fold in the DM $+C P$ group - significantly suppressed $(n=3$, $P<0.05)$ (Fig. 2B and C). In addition, there were consistent differences among the groups in COL4 distribution and content (Fig. 3A and B). Compared with the Con group $(95.33 \pm 9.45)$, distinct COL4 staining was observed in the DM group $(143.33 \pm 11.59)(P<0.01)$, which was attenuated in the DM+CP group $(106.00 \pm 13.52)(P<0.05)$.

The effects of C-peptide on renal TGFB1 and SMAD3 protein were also assessed in diabetic rats at 12 weeks (Fig. 3C). Compared with the Con group, both TGFB1 and SMAD3 protein contents were $3.15 \pm 0.42$ and $1.46 \pm 0.14$ fold, significantly more abundant in the DM group, but only the amount of TGFB1 protein was $1.93 \pm 0.31$-fold, lower in the $\mathrm{DM}+\mathrm{CP}$ group $(n=3, P<0.05$ or 0.01$)($ Fig. $3 \mathrm{D}$

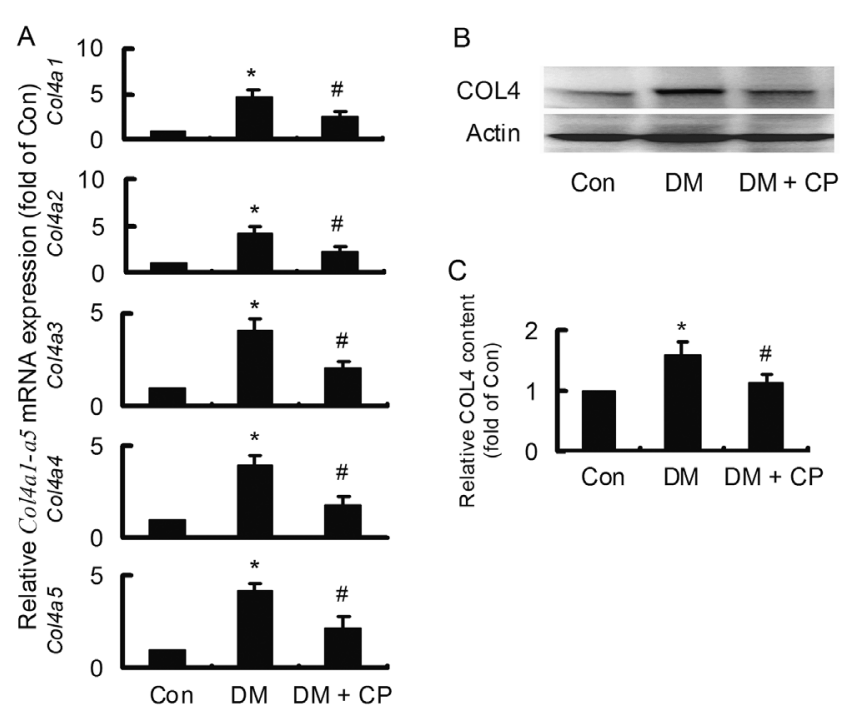

Figure 2

C-peptide suppressed COL4 generation in diabetic nephropathy. (A) The mRNA expression of Col4a1-a5 was detected by real-time RT-PCR. The protein content of COL4 was detected by western blotting (B) and calculated (C). ${ }^{\star} P<0.05$ vs control (Con) group; $\#<0.05$ vs $D M$ group $(n=3)$. CP, C-peptide; DM, diabetes mellitus.

and $\mathrm{E}$ ). In addition, C-peptide had a tendency to inhibit the increased phospho-SMAD3 contents, but without significant difference (Supplementary Fig. 3A and B).

These results suggest that C-peptide can suppress renal Col4a1-a5 mRNA expression and reduce COL4 protein level, alongside a reduction in TGFB1 protein content, in this rat model of DM.

\section{C-peptide inhibits HG-stimulated COL4 generation in mesangial cells}

The effects of HG on Col4a1-a5 mRNA expression, and COL4 protein and secretion, were initially measured. Compared with the $5 \mathrm{mM}$ group, expressions of Col4a1-a5 were increased after 24-h HG stimulation, especially $4.12 \pm 1.05, \quad 4.01 \pm 1.04, \quad 3.83 \pm 0.92, \quad 4.32 \pm 1.20$ and $3.56 \pm 0.75$-fold at $35 \mathrm{mM}(n=3, P<0.05$ or 0.01$)$ (Fig. $4 \mathrm{~A})$. HG had similar effects on COL4 protein content and secretion at $48 \mathrm{~h}$, which were $1.63 \pm 0.14$ and $1.79 \pm 0.26$ fold at $35 \mathrm{mM}(n=3, P<0.05$ or 0.01$)$ (Fig. $4 \mathrm{~B}$ and $\mathrm{C})$, indicating that COL4 generation can be stimulated by HG.

Then, the concentration-dependent effects of C-peptide on Col4a1-a5 mRNA expression, COL4 protein and secretion were investigated in HG-stimulated mesangial cells. Compared with the LG group, mRNA expressions of Col4a1-a5 were $4.11 \pm 1.05,4.64 \pm 0.95,3.98 \pm 0.77$, $4.32 \pm 0.93$ and $3.64 \pm 0.93$-fold, significantly higher in 
A

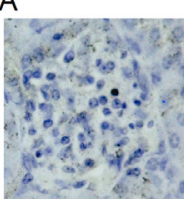

Con

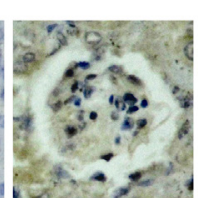

DM

B

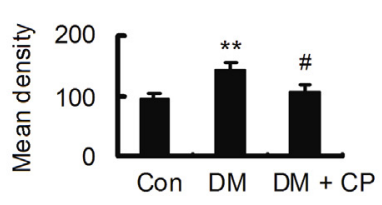

C

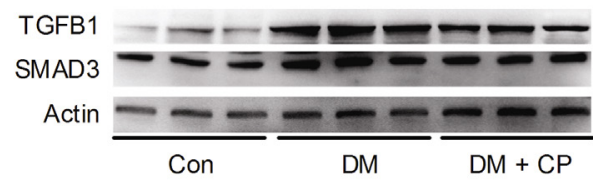

$\mathrm{D}$

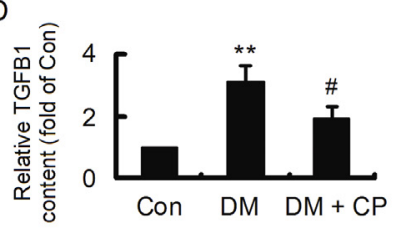

E

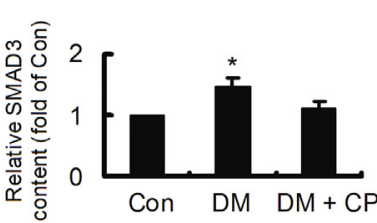

Figure 3

C-peptide suppressed COL4 distribution and TGFB1 content in diabetic nephropathy. The COL4 distribution was illustrated by immunohistochemistry (A) and calculated (B). (C) The protein contents of renal TGFB1 and SMAD3 were detected by western blotting. (D) TGFB1 content was obviously higher in DM group, but was significantly inhibited by C-peptide. (E) The increased SMAD3 content was not significantly inhibited by C-peptide. ${ }^{*} P<0.05$ or ${ }^{*} P<0.01$ vs control (Con) group; ${ }^{\#}<0.05$ vs DM group $(n=3)$. CP, C-peptide; DM, diabetes mellitus.

the HG group $(n=3, P<0.01)$, but only slightly higher in the L-HG group at $24 \mathrm{~h}$. The HG-stimulated increases in mRNA expressions of Col4a1-a5 were 1.29 \pm 0.39 , $1.56 \pm 0.45,1.58 \pm 0.49,1.52 \pm 0.31$ and $1.10 \pm 0.31$-fold in the presence of $0.5 \mathrm{nM}$ C-peptide and $1.70 \pm 0.46$, $1.90 \pm 0.52,2.14 \pm 0.51,2.06 \pm 0.60$ and $1.33 \pm 0.89$-fold in the presence of $0.9 \mathrm{nM} \mathrm{C}$-peptide, substantially suppressed at $24 \mathrm{~h}(n=3, P<0.01)$ (Fig. 5A). Compared with the LG group, the HG-stimulated $2.20 \pm 0.16$ and $1.69 \pm 0.15$ fold increases in COL4 protein content and secretion were inhibited by C-peptide treatment at $48 \mathrm{~h}$, especially $1.71 \pm 0.25$ and $1.21 \pm 0.12$-fold at $0.5 \mathrm{nM}(n=3, P<0.05$ or 0.01 ) (Fig. $5 \mathrm{~B}$ and $\mathrm{C}$ ), consistent with the changes in Col4a1-a5 mRNA expression.

These results demonstrate that C-peptide can inhibit COL4 generation in HG-stimulated mesangial cells in a concentration-dependent manner. Furthermore, at its physiological concentration $(0.5 \mathrm{nM})$, C-peptide had its most conspicuous effect, and this concentration was therefore adopted for subsequent experiments.

\section{C-peptide has no discernible effect on HG-stimulated SMAD3 nuclear translocation}

First, the effects of C-peptide on TGFB1 and TSP-1 proteins were investigated in $\mathrm{HG}$-stimulated mesangial cells. Compared with the LG group, the HG-induced $1.62 \pm 0.24$ and $1.66 \pm 0.17$-fold increase in TGFB1 and TSP-1 protein contents were reduced significantly by C-peptide to $1.11 \pm 0.18$ and $1.10 \pm 0.23$-fold at $48 \mathrm{~h}(n=3$, $P<0.05$ ) (Fig. 6A).

Subsequently, the nuclear translocation of SMAD3 was assessed using western blotting (Fig. 6B) by measuring the ratio of nuclear SMAD3 to its total content (Fig. 6C). Compared with the LG group, nuclear SMAD3 content in the $\mathrm{HG}$ and $\mathrm{HG}+\mathrm{CP}$ groups was $2.00 \pm 0.37$ and $1.69 \pm 0.28$-fold, significantly higher at $48 \mathrm{~h}(n=3, P<0.05)$. However, no significant difference was observed between the $\mathrm{HG}$ and $\mathrm{HG}+\mathrm{CP}$ groups. Besides, C-peptide had a tendency to inhibit the HG-induced increase of phosphoSMAD3 contents, but without significant difference (Supplementary Fig. 3C).

Furthermore, SMAD3 nuclear translocation was visualized using immunofluorescence (Fig. 7A). Immunofluorescent staining for SMAD3 showed that it was scattered throughout the cytoplasm in the LG group, but was mainly within the nucleus in the HG group. The HG-stimulated predominantly nuclear distribution of SMAD3 was not clearly affected by C-peptide treatment at $24 \mathrm{~h}$. And consistently, the percentage of SMAD3 nuclear

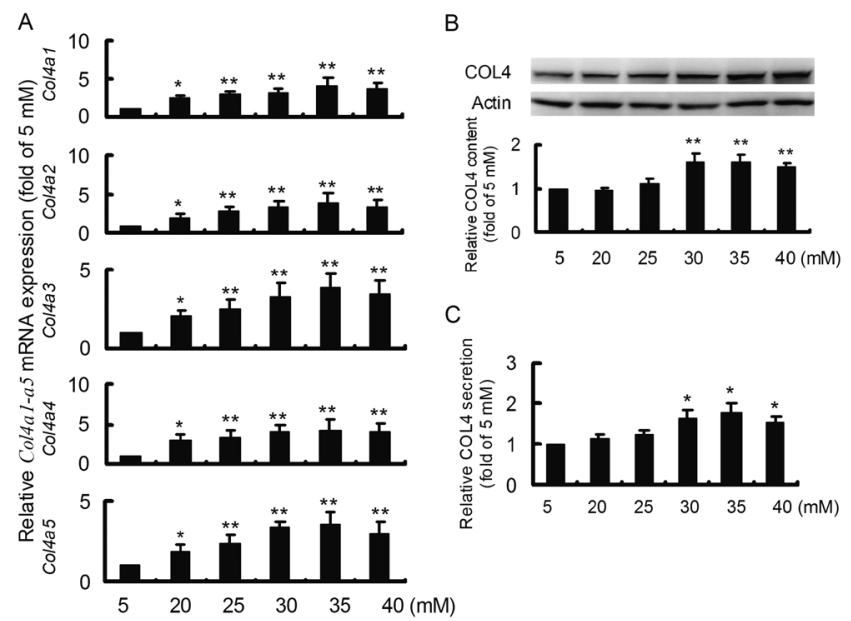

Figure 4

Effects of high glucose (HG) on COL4 generation in mesangial cells. After the cells were stimulated with different concentrations of HG, mRNA expression of Col4a1-a5 was detected by real-time RT-PCR (A), COL4 protein content by western blotting (B) and COL4 secretion by ELISA (C). ${ }^{*} P<0.05$ or $* * P<0.01$ vs $5 \mathrm{mM}$ group. The data were repeated three times and represented as mean \pm S.D. 


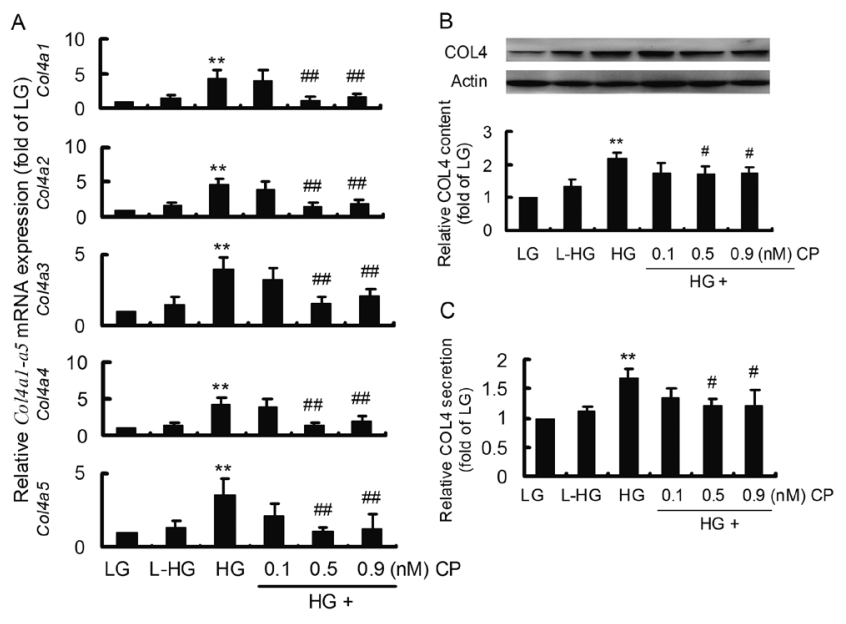

Figure 5

C-peptide inhibited COL4 generation in high glucose (HG)-stimulated mesangial cells. After the cells were incubated with C-peptide at different concentrations, mRNA expression of Col4a1-a5 was detected by real-time RT-PCR (A), COL4 protein content by western blotting (B), and COL4 secretion by ELISA (C). $* * P<0.01$ vs Low glucose (LG) group; ${ }^{*} P<0.05$ or $\# P<0.01$ vs HG group. The data were repeated three times and represented as mean \pm s.D. CP, C-peptide; L-HG, high L-glucose.

translocation was also elevated in the $\mathrm{HG}$ and $\mathrm{HG}+\mathrm{CP}$ groups (Fig. 7B).

These results imply that although it reduces TGFB1 protein content, C-peptide does not seem to have an effect on HG-stimulated nuclear translocation of SMAD3 in mesangial cells.

\section{C-peptide prevents SMAD3 from binding to Col4a promoters in HG-stimulated mesangial cells}

Immunofluorescence showed that C-peptide could enter cell and concentrated in nucleus for HG stimulation (Supplementary Fig. 1). Based on the nuclear localization of C-peptide, the binding of SMAD3 to its cognate sites in the Col4a1a2, Col4a3a4 and Col4a5 promoters was measured using ChIP (Fig. 8). Compared with the LG group, the quantity of SMAD3-bound site in the Col4a1a2 promoters was significantly higher in the HG group, but this was significantly lower in the $\mathrm{HG}+\mathrm{CP}$ group at $24 \mathrm{~h}$ (Fig. 8A). The HG-stimulated increases in SMAD3-bound sites in the Col4a3a4 and Col4a5 promoters were also significantly inhibited by C-peptide treatment (Fig. 8B and C).

Meanwhile, the contents of SMAD3 bound to the Col4a1a2, Col4a3a4 and Col4a5 promoters were measured by DNA pull-down assay (Fig. 9). The content of SMAD3 bound to the Col4a1a2 promoters was increased in the HG group, but was significantly reduced by C-peptide treatment at 24 h (Fig. 9A). Similarly, C-peptide treatment
A

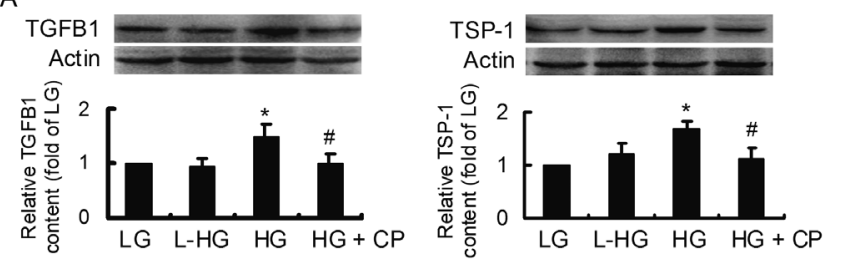

B

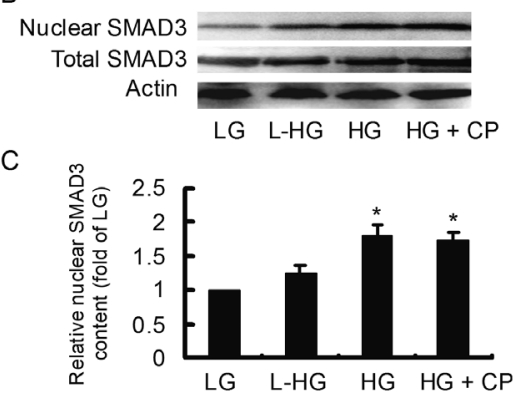

Figure 6

Effects of C-peptide on TGFB1 and SMAD3 protein in high glucose (HG)-stimulated mesangial cells. After the cells were treated with $0.5 \mathrm{nM}$ C-peptide, TGFB1 and TSP-1 protein contents (A) and total and nuclear contents of SMAD3 (B) were detected by western blotting. (C) The ratio of nuclear SMAD3 to total SMAD3 was calculated to show SMAD3 nuclear translocation. ${ }^{*} P<0.05$ vs low glucose $(\mathrm{LG})$ group; ${ }^{*} P<0.05$ vs HG group. The data were repeated three times and represented as mean \pm S.D. CP, C-peptide; L-HG, high L-glucose.

also reduced the HG-increased SMAD3 content in Col4a3a4 and Col4a5 promoters with significant difference (Fig. 9B and C).

These results suggest that C-peptide may prevent the HG-stimulated binding of SMAD3 to its sites in the Col4a1a2, Col4a3a4 and Col4a5 promoters, resulting in the inhibition of Col4a1-5 expression in mesangial cells.

\section{Discussion}

Although C-peptide is known to attenuate ECM accumulation and therefore have beneficial effects in DN, the underlying mechanism has not been fully elucidated. Activation of TGFB1/SMAD3 signaling pathway may lead to excess production of COL4, the major contributor to ECM accumulation in DN. However, whether and how C-peptide affects TGFB1/SMAD3-stimulated COL4 generation is unclear. In the study, we have shown that, in addition to reducing TGFB1 content, C-peptide can prevent SMAD3 from binding to its cognate sites in the Col4a1a2, Col4a3a4 and Col4a5 promoters, thereby suppressing COL4 generation in vivo and in vitro. We propose this as a mechanism whereby C-peptide attenuates ECM accumulation in DN.

TGFB1 promotes ECM production and simultaneously inhibits its degradation, resulting in progressive renal 
A

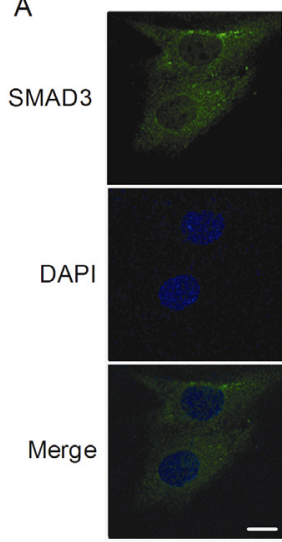

LG

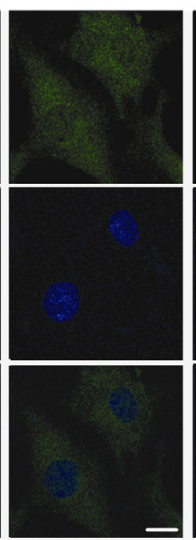

L-HG

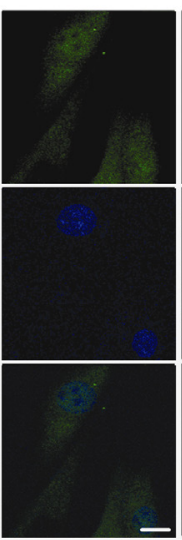

HG

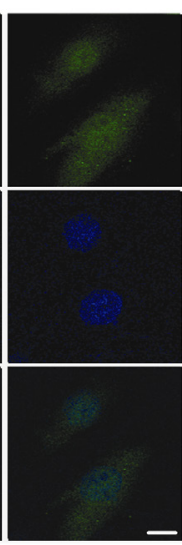

$\mathrm{HG}+\mathrm{CP}$
B

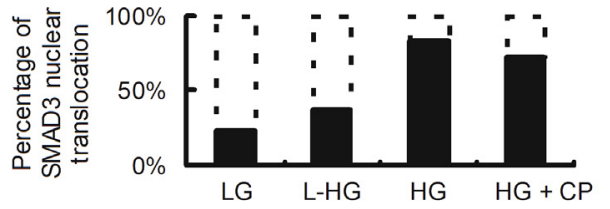

Figure 7

Effects of C-peptide on SMAD3 nuclear translocation in high glucose (HG)-stimulated mesangial cells. (A) The nuclear translocation of SMAD3 was illustrated by immunofluorescence. Scale bar $10 \mu \mathrm{m}$. (B) The percentage of SMAD3 nuclear translocation was calculated. CP, C-peptide; L-HG, high L-glucose.

fibrosis (Meng etal. 2016). In this study, we have also shown that TGFB1 protein levels were higher in both in vivo and in vitro models. Activation of TGFB1/SMAD3 signaling causes production of excess COL4, which is considered to be the central pathogenic process contributing to ECM accumulation in DN (Gu et al. 2016). However, because TGFB1 signaling regulates various cellular processes, its inhibition could lead to undesirable side effects (Yiang et al. 2016), and thefore there is still no effective therapy for ECM accumulation in DN.

C-peptide has been shown to have unique beneficial effects in DN (Hills et al. 2010a,b, Xu et al. 2015), specifically the attenuation of ECM accumulation and fibrosis (Hills et al. 2010a,b, Brunskill 2017, Wahren 2017). In this study, the effects of C-peptide on COL4 generation were investigated in vivo and in vitro. First, it was found that C-peptide could attenuate glomerular ECM accumulation, reduce Col4a1-a5 mRNA expression and COL4 protein content in a diabetic rat model, without having an effect on blood glucose. In addition, C-peptide suppressed TGFB1 content in this model. Second, C-peptide had concentration-dependent effects to inhibit Col4a1-a5 mRNA expression, and COL4 protein content and secretion, in HG-stimulated mesangial cells. C-peptide had its most conspicuous effect at its
A

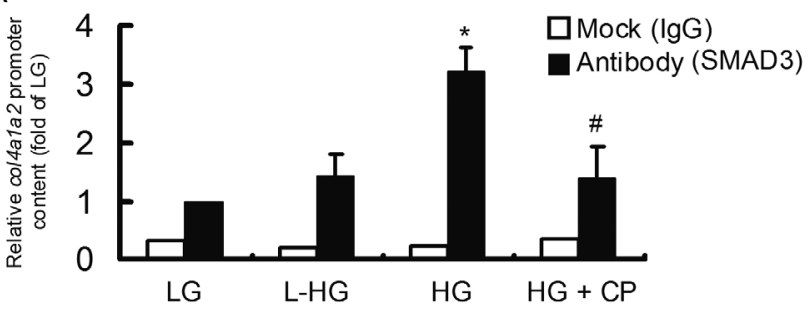

B

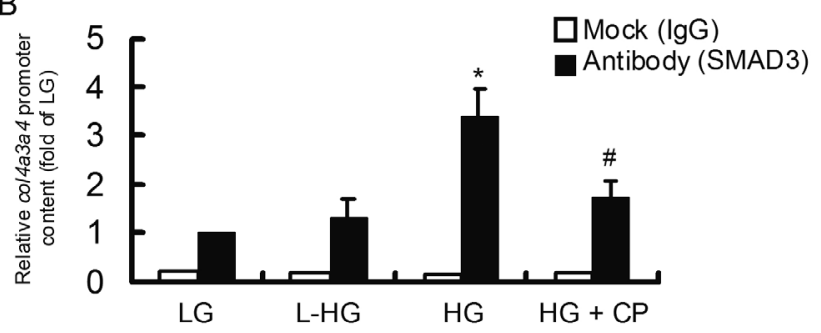

C

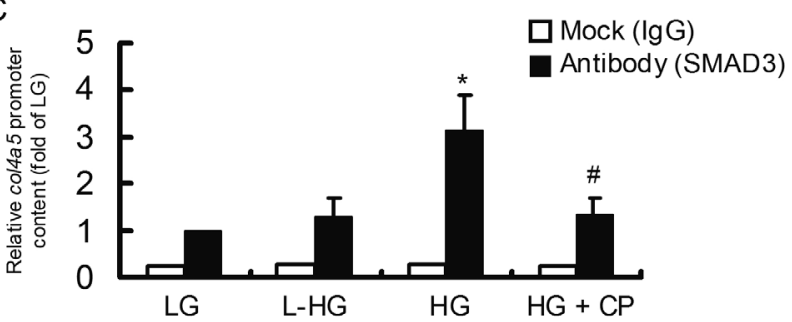

Figure 8

C-peptide prevented SMAD3 from binding to its sites in high glucose (HG)-stimulated mesangial cells. The bindings of SMAD3 to its sites in the Col4a1a2 promoter (A), Col4a3a4 promoter (B), and Col4a5 promoter (C) were detected by ChIP. ${ }^{*} P<0.05$ vs low glucose (LG) group; ${ }^{\#} P<0.05$ vs HG group. The data were repeated three times and represented as mean \pm S.D. CP, C-peptide; L-HG, high L-glucose.

physiological concentration, indicating that it may have a physiologically relevant effect on excess COL4 synthesis in DN. However, the effect of C-peptide on TGFB1/SMAD3activated COL4 generation remains to be evaluated.

Several mechanisms have been reported for the protective effects of $\mathrm{C}$-peptide, including receptor bindingmediated effects, interaction with proteins to affect their function, and localization to the nucleus to regulate gene expression (Lindahl et al. 2010, Garcia-Serrano et al. 2015, Kolar et al. 2017). However, these mechanisms are not specific to DN and do not at present explain the unique effects of C-peptide to attenuate ECM accumulation in DN. We have previously shown that C-peptide scarcely enters mesangial cells when they are cultured in medium containing a normal glucose concentration, but in the presence of $\mathrm{HG}, \mathrm{C}$-peptide might be internalized via activated endocytosis and dynamically localizes to the nucleus, exhibiting its protective mechanism (Li et al. $2013 b$ ). In this study, the effects of C-peptide on TGFB1 
A
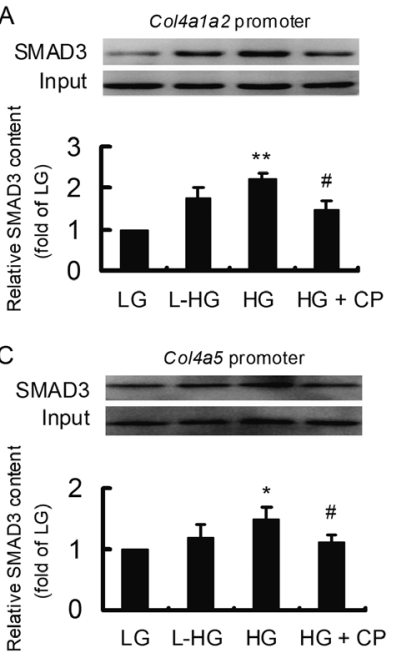

Figure 9

C-peptide reduced SMAD3 bound to its sites in high glucose (HG)stimulated mesangial cells. The contents of SMAD3 bound to the Col4a1a2 promoter (A), Col4a3a4 promoter (B) and Col4a5 promoter (C) were analyzed by DNA pull-down assay. ${ }^{*} P<0.05$ or $* * P<0.01$ vs low glucose (LG) group; $\# P<0.05$ vs HG group. The data were repeated three times and represented as mean \pm s.D. CP, C-peptide.

protein content and SMAD3 nuclear translocation were measured in HG-stimulated mesangial cells. Although C-peptide reduced TGFB1 protein content, it did not reduce HG-stimulated nuclear SMAD3 content in mesangial cells. This phenomenon may be attributed to the nuclear localization of C-peptide to regulate gene expressions, including COL4 and even TGFB1.

Then, the HG-stimulated binding of SMAD3 to its cognate sites in the Col4a1a2, Col4a3a4 and Col4a5 promoters were also measured, and this showed that C-peptide can prevent such bindings, thereby inhibiting Col4a1-5 mRNA expression. Lindahl et al. also reported that C-peptide could bind to histones to regulate gene expression (Lindahl et al. 2010). From the results of current study, it is speculated that the effect of C-peptide might be mediated by its transport to the nucleus, where it interacts with SMAD3 and/or histones to suppress the binding of SMAD3 to Col4a promoters, regulating Col4a15 expressions. However, this needs further investigation in the future study, with a scrambled control to specify the mechanism of C-peptide.

This study has added information to our knowledge of the mechanism whereby C-peptide can suppress TGFB1/ SMAD3-stimulated COL4 synthesis in DN. These findings may provide a basis for the development of more effective and specific treatments for ECM accumulation in DN. Additional work should be aimed at identifying the particular region of the peptide sequence responsible for its effect.
In conclusion, C-peptide can prevent SMAD3 from binding to its sites in the Col4a1a2, Col4a3a4 and Col4a5 promoters, suppress Col4a1-a5 mRNA expressions and reduce COL4 synthesis in vivo and in vitro. Our results provide additional details of the mechanism whereby C-peptide alleviates ECM accumulation in DN.

\section{Supplementary data}

This is linked to the online version of the paper at https://doi.org/10.1530/ JME-18-0009.

Declaration of interest

The authors declare that there is no conflict of interest that could be perceived as prejudicing the impartiality of the research reported.

\section{Funding}

This work was supported by Grants from the National Natural Science Foundation of China (81070658 and 81470595) and the Hebei Natural Science Foundation (H2015206101).

\section{Author contribution statement}

$J \mathrm{Q}$ designed the experiments. $\mathrm{Y} L$ and $\mathrm{Y} Z$ performed the in vivo experiments. $\mathrm{Y} \mathrm{L}, \mathrm{W} \mathrm{G}$ and $\mathrm{X} \mathrm{G}$ performed in vitro experiments and made the figure. $Y Z, H Q$ and $K L$ performed the statistics and made the remaining figures. J $Q$ wrote the manuscript and all authors reviewed and edited the manuscript.

\section{Acknowledgments}

The authors thank Mark Cleasby, PhD, from Liwen Bianji, Edanz Group China (www.liwenbianji.cn/ac), for editing the English text of a draft of this manuscript.

\section{References}

Al-Waili N, Al-Waili H, Al-Waili T \& Salom K 2017 Natural antioxidants in the treatment and prevention of diabetic nephropathy; a potential approach that warrants clinical trials. Redox Report 9 1-20. (https://doi.org/10.1080/13510002.2017.1297885)

Brunskill NJ 2017 C-peptide and diabetic kidney disease. Journal of Internal Medicine 281 41-51. (https://doi.org/10.1111/joim.12548)

Chung AC, Huang XR, Meng X \& Lan HY 2010 miR-192 mediates TGFbeta/Smad3-driven renal fibrosis. Journal of the American Society of Nephrology 21 1317-1325. (https://doi.org/10.1681/ASN.2010020134)

Dimas GG, Didangelos TP \& Grekas DM 2017 Matrix gelatinases in atherosclerosis and diabetic nephropathy: progress and challenges. Current Vascular Pharmacology 15 557-565. (https://doi.org/10.2174/1 $570161115666170202162345)$

Donegan D, Bale LK \& Conover CA 2016 PAPP-A in normal human mesangial cells: effect of inflammation and factors related to diabetic nephropathy. Journal of Endocrinology 231 71-80. (https://doi. org/10.1530/JOE-16-0205)

Fan Y, Zhang J, Xiao W, Lee K, Li Z, Wen J, He L, Gui D, Xue R, Jian G, et al. 2017 Rtn1a-mediated endoplasmic reticulum stress in podocyte 
injury and diabetic nephropathy. Scientific Reports 7 323. (https://doi. org/10.1038/s41598-017-00305-6)

Flyvbjerg A 2017 The role of the complement system in diabetic nephropathy. Nature Reviews Nephrology 13 311-318. (https://doi. org/10.1038/nrneph.2017.31)

Gao P, Wei Y, Zhang Z, Zeng W, Sun D, Liu D, Hou B, Zhang C, Zhang N, Li H, et al. 2016 Synergistic effects of c-Jun and SP1 in the promotion of TGF $\beta 1$-mediated diabetic nephropathy progression. Experimental and Molecular Pathology 100 441-450. (https://doi. org/10.1016/j.yexmp.2016.04.005)

Garcia-Serrano S, Gutiérrez-Repiso C, Gonzalo M, Garcia-Arnes J, Valdes S, Soriguer F, Perez-Valero V, Alaminos-Castillo MA, Francisco Cobos-Bravo J, Moreno-Ruiz FJ, et al. 2015 C-peptide modifies leptin and visfatin secretion in human adipose tissue. Obesity $\mathbf{2 3}$ 1607-1615. (https://doi.org/10.1002/oby.21137)

Gu C, Zhang J, Noble NA, Peng XR \& Huang Y 2016 An additive effect of anti-PAI-1 antibody to ACE inhibitor on slowing the progression of diabetic kidney disease. American Journal of Physiology: Renal Physiology 311 F852-F863. (https://doi.org/10.1152/ ajprenal.00564.2015)

Hills CE, Brunskill NJ \& Squires PE 2010a C-peptide as a therapeutic tool in diabetic nephropathy. American Journal of Nephrology 31 389-397. (https://doi.org/10.1159/000289864)

Hills CE, Willars GB \& Brunskill NJ $2010 b$ Proinsulin C-peptide antagonizes the profibrotic effects of TGF-beta1 via up-regulation of retinoic acid and HGF-related signaling pathways. Molecular Endocrinology 24 822-831. (https://doi.org/10.1210/me.2009-0391)

Ido Y, Vindigni A, Chang K, Stramm L, Chance R, Heath WF, DiMarchi RD, Di Cera E \& Williamson JR 1997 Prevention of vascular and neural dysfunction in diabetic rats by C-peptide. Science 277 563-566. (https://doi.org/10.1126/science.277.5325.563)

Kato M, Wang M, Chen Z, Bhatt K, Oh HJ, Lanting L, Deshpande S, Jia Y, Lai JY, O'Connor CL, et al. 2016 An endoplasmic reticulum stress-regulated lncRNA hosting a microRNA megacluster induces early features of diabetic nephropathy. Nature Communications $\mathbf{7}$ 12864. (https://doi.org/10.1038/ncomms12864)

Kolar GR, Grote SM \& Yosten GL 2017 Targeting orphan G proteincoupled receptors for the treatment of diabetes and its complications: C-peptide and GPR146. Journal of Internal Medicine 281 25-40. (https://doi.org/10.1111/joim.12528)

Li Y, Liu D, Liu Y, Li E, Wang H, Liu K \& Qi J 2013a Protein nitration promotes inducible nitric oxide synthase transcription mediated by NF-kB in high glucose-stimulated human lens epithelial cells. Molecular and Cellular Endocrinology 370 78-86. (https://doi. org/10.1016/j.mce.2013.02.015)

Li Y, Zhao M, Li B \& Qi J 2013b Dynamic localization and functional implications of C-peptide might for suppression of iNOS in high glucose-stimulated rat mesangial cells. Molecular and Cellular Endocrinology 381 255-260. (https://doi.org/10.1016/j. mce.2013.08.007)

Li Y, Liu D, Zong Y, Qi J, Li B, Liu K \& Xiao H 2015 Developmental stage-specific hepatocytes induce maturation of HepG2 cells by rebuilding the regulatory circuit. Molecular Medicine 21 285-295. (https://doi.org/10.2119/molmed.2014.00173)

Li B, Li Y, Liu K, Wang X, Qi J, Wang B \& Wang Y 2017 High glucose decreases claudins -5 and -11 in cardiac microvascular endothelial cells: Antagonistic effects of tongxinluo. Endocrine Research 42 15-21. (https://doi.org/10.3109/07435800.2016.1163723)
Lindahl E, Nyman U, Zaman F, Palmberg C, Cascante A, Shafqat J, Takigawa M, Sävendahl L, Jörnvall H \& Joseph B 2010 Proinsulin C-peptide regulates ribosomal RNA expression. Journal of Biological Chemistry 285 3462-3469. (https://doi.org/10.1074/jbc. M109.053587)

Marshall CB 2016 Rethinking glomerular basement membrane thickening in diabetic nephropathy: adaptive or pathogenic? American Journal of Physiology: Renal Physiology 311 F831-F843. (https://doi.org/10.1152/ajprenal.00313.2016)

Meng XM, Nikolic-Paterson DJ \& Lan HY 2016 TGF- $\beta$ : the master regulator of fibrosis. Nature Reviews Nephrology 12 325-338. (https:// doi.org/10.1038/nrneph.2016.48)

Peng FF, Xiao ZL, Chen HM, Chen Y, Zhou J, Yu H \& Zhang BF 2016 Parathyroid hormone inhibits TGF- $\beta /$ Smad signaling and extracellular matrix proteins upregulation in rat mesangial cells. Biochemical and Biophysical Research Communications 478 1093-1098. (https://doi.org/10.1016/j.bbrc.2016.08.073)

Robertson WE, Rose KL, Hudson BG \& Vanacore RM 2014 Supramolecular organization of the $\alpha 121-\alpha 565$ collagen IV network. Journal of Biological Chemistry 289 25601-25610. (https://doi. org/10.1074/jbc.M114.571844)

Shaw JA, Shetty P, Burns KD, Fergusson D \& Knoll GA 2015 C-peptide as a therapy for kidney disease: a systematic review and meta-analysis. PLOS ONE 10 e0127439. (https://doi.org/10.1371/journal. pone.0127439)

Sheu ML, Shen CC, Jheng JR \& Chiang CK 2017 Activation of PI3K in response to high glucose leads to regulation of SOCS-3 and STAT1/3 signals and induction of glomerular mesangial extracellular matrix formation. Oncotarget 8 16925-16938. (https://doi.org/10.18632/ oncotarget.14808)

Sugiyama H, Tokunaka K, Hayashi T, Imamura Y, Morita M \& Yamato M 2015 Non-triple helical form of type IV collagen $\alpha 1$ chain. Heliyon $\mathbf{1}$ e00051. (https://doi.org/10.1016/j.heliyon.2015.e00051)

Sun W, Gao X, Zhao X, Cui D \& Xia Q 2010 Beneficial effects of C-peptide on renal morphology in diabetic rats. Acta Biochimica et Biophysica Sinica 42 893-899. (https://doi.org/10.1093/abbs/gmq100)

Turner AW, Nikpay M, Silva A, Lau P, Martinuk A, Linseman TA, Soubeyrand S \& McPherson R 2015 Functional interaction between COL4A1/COL4A2 and SMAD3 risk loci for coronary artery disease. Atherosclerosis 242 543-552. (https://doi.org/10.1016/j. atherosclerosis.2015.08.008)

Wahren J 2017 C-peptide and the pathophysiology of microvascular complications of diabetes. Journal of Internal Medicine 281 3-6. (https://doi.org/10.1111/joim.12541)

Wahren J \& Larsson C 2015 C-peptide: new findings and therapeutic possibilities. Diabetes Research and Clinical Practice 107 309-319. (https://doi.org/10.1016/j.diabres.2015.01.016)

Xu S, Jiang Y, Wang H, Wang Z, Liu H, Peng L, Fang Q, Deng T, You J, Zhou $\mathrm{X}$, et al. $2015 \mathrm{C}$-peptide ameliorates renal injury in type 2 diabetic rats through protein kinase A-mediated inhibition of fibronectin synthesis. Biochemical and Biophysical Research Communications 458 674-680. (https://doi.org/10.1016/j. bbrc.2015.02.022)

Yiang GT, Chen JN, Lin PS, Liu HC, Chen SY \& Wei CW 2016 Combined treatment with vitamin $\mathrm{E}$ and gefitinib has synergistic effects to inhibit TGF- $\beta 1$-induced renal fibroblast proliferation. Molecular Medicine Reports 13 5372-5378. (https://doi.org/10.3892/ mmr.2016.5155)

Received in final form 8 April 2018

Accepted 2 May 2018

Accepted Preprint published online 2 May 2018 (c) 2018 Society for Endocrinology Published by Bioscientifica Ltd. Printed in Great Britain 